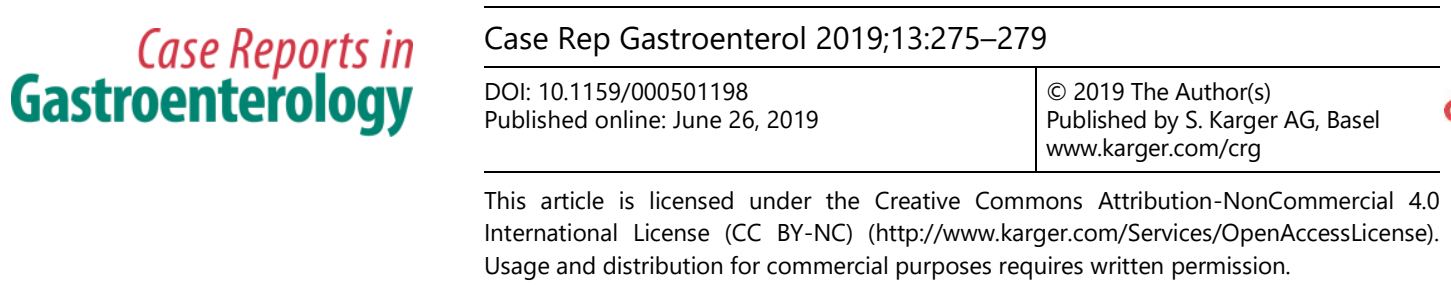

\title{
Transplant-Amenable Hepatocellular Carcinoma in a Fontan Patient
}

\author{
Brian Patrick Rutledge $^{a} \quad$ Anupama Devara $^{a} \quad$ Sindhuri Benjaram ${ }^{a}$ \\ Stephanie Judd ${ }^{a} b \quad$ Murray Ehrinpreis ${ }^{a}$ \\ aWayne State University School of Medicine, Detroit, MI, USA; bJohn D. Dingell VA \\ Medical Center, Detroit, MI, USA
}

\section{Keywords}

Fontan procedure $\cdot$ Cirrhosis $\cdot$ Hepatocellular carcinoma $\cdot$ Liver disease $\cdot$ Congenital heart disease

\begin{abstract}
The Fontan circulation alters a patient's physiology and imparts long-term risks related to chronically elevated systemic venous pressure. An increasing number of patients with Fontan physiology are surviving into adulthood and are at risk of hepatic sequalae. The ideal timeline and method of hepatic surveillance in the Fontan population remains to be defined. In this case, the patient was diagnosed with hepatocellular carcinoma more than 20 years after undergoing the Fontan procedure and was a candidate for combined heart-liver transplant. That her disease progressed prior to organ availability supports the argument for hepatic surveillance in this population.

(C) 2019 The Author(s)

Published by S. Karger AG, Basel
\end{abstract}

\section{Introduction}

Single ventricular anatomy is rare, occurring in approximately 120 out of every 100,000 live births [1]. These individuals are living well into adulthood because of the Fontan procedure, the definitive palliative intervention for univentricular cardiac anatomy [2]. A notable physiologic consequence to this intervention is chronic systemic elevation of venous pressure $[2,3]$, represented in the liver as passive venous congestion, which is a risk factor for hepatic 


\section{Case Reports in Gastroenterology}

Case Rep Gastroenterol 2019;13:275-279

DOI: 10.1159/000501198

(c) 2019 The Author(s). Published by S. Karger AG, Basel www.karger.com/crg

Rutledge et al.: Transplant-Amenable Hepatocellular Carcinoma in a Fontan Patient

dysfunction that may eventually lead to hepatic fibrosis, cirrhosis, and hepatocellular carcinoma (HCC) [4]. Favorable cardiac outcomes from the procedure have created a growing adult cohort that is at risk for long-term hepatic complications from the altered circulation $[2,3]$.

\section{Case Presentation}

A 35-year-old female with history of complex univentricular anatomy underwent classic atriopulmonary Fontan procedure in early childhood. She had a complicated course that included multiple surgical revisions, arrhythmias necessitating pacemaker placement, and multiple thrombectomies including a right atrial thrombus evacuation. Approximately 20 years following the initial Fontan procedure, the patient developed clinical hepatomegaly. Hepatitis $\mathrm{B}$ and $\mathrm{C}$ serologies were negative, and there was no history of excess alcohol use. She had not been under any hepatic surveillance and did not undergo any hepatic imaging until nearly 3 years later. A CT with contrast was performed at that time in 2011 and showed cirrhotic features. Additionally, several liver lesions were found with a dominant lesion which was felt to be most likely related to adenoma (Fig. 1). However, an alpha-fetoprotein level of 564 raised the suspicion for HCC, and therefore the diagnosis was definitively obtained by biopsy at an outside hospital which showed HCC.

The patient subsequently qualified for placement on the waiting list for combined heart and liver transplant (CHLT). Unfortunately, during the interim waiting period, she developed an extrahepatic lesion, which was also confirmed by biopsy to be secondary to the HCC primary lesion. The plan for CHLT transplantation was abandoned. Due to the presence of a cardiac pacemaker, radiofrequency ablation was not attempted. The patient did have multiple treatments with stereotactic body radiation for both intra- and extrahepatic lesions. When another focus of the tumor in the liver developed, an attempt at chemoembolization was made but had to be cancelled after being complicated by an episode of cardiac arrest. Ultimately, the disease continued to progress with development of peritoneal metastases, and it eventually led to her death.

\section{Discussion}

This case is best viewed from its illustrative potential. Over 20 years after undergoing the Fontan procedure and 3 years after the onset of hepatomegaly, the patient was found to have transplant-amenable HCC. The difficult question that arises in review of this case is whether a successful CHLT operation might have been undertaken if the patient was monitored in a hepatic surveillance imaging protocol. The clinical course suggests that surveillance imaging may have led to more prompt placement on the transplant list. Although CHLT operations are rare and limited to select centers, it has been shown they can be performed with excellent outcomes in both short and long-term follow-up in patients with Fontan physiology [5].

The Fontan procedure has undergone many modifications since its inception, but the underlying principle remains the same: direction of blood flow from the systemic venous side passively to the pulmonary arteries without passage through a functional ventricle [2]. Success in managing the long-term cardiac manifestations of the congenital univentricular heart disease with this procedure has improved survival [2]. This has created a surviving patient cohort at risk of long-term hepatic manifestations [3]. The underlying reason for this risk relates to the chronic low cardiac output with accompanying high venous pressures [4]. When 
the increased pressure occurs, it is typically continuous in nature and causes progressive damage over time, a probable explanation for the strong association with hepatic dysfunction and cirrhosis [3]. Risk stratification has been performed in a variety of patient subsets including hepatitis B and C cohorts with and without cirrhosis as well as the general population. In contrast, it is comparatively uncommon to find HCC surveillance guidelines regarding a cardiac cause. Although it is now gaining traction within the field of Cardiology, the topic continues to be of limited consideration within the field of Gastroenterology [6, 7]. Outside of congenital heart disease, the association between heart disease and HCC is rarely observed [8]. Yet, as the Fontan cohort ages, it has become evident that the burden of liver disease is significant in this subset of cardiac patients [2]. The importance of surveillance, as suggested by this case, is the potential for early identification of patients with potential to be placed on the CHLT list. This is important as the amount of time spent waiting on the CHLT list can be significant and is associated with a greater risk of wait-list mortality than with single-organ transplants [9].

Surveillance guidelines for Fontan patients are an ongoing topic of debate and discussion $[2,3,6,10]$. A primary focus of research relates to devising a standardized noninvasive modality to assess for liver fibrosis and cirrhosis [10]. There are currently no official guidelines related to the use of serum markers in this capacity [10]. Elastography is often used to noninvasively measure liver stiffness, but in those with underlying hepatic congestion, such as in the Fontan population, an overestimation of liver stiffness is seen [11]. More promising is shear wave sonoelastography, which has shown a strong correlation between assessed stiffness and histology but is not yet standardized [12]. Some centers utilize hepatic magnetic resonance elastography (MRE) to assess for both fibrosis and HCC in conjunction with the cardiac MRI's often performed in this cohort [3,13]. MRE is positively correlated with many measures of liver disease progression (estimated stiffness, AST to platelet ratio (APRI) index, model for end stage liver disease (MELD) score, pressures in the Fontan conduit, histological damage); however, it is limited by its cost and lack of widespread availability [11,13]. Presently, annual ultrasound screening is the standard unless and until there is confirmed cirrhosis at which time MRI becomes the preferred modality [10]. Early initiation of a screening protocol, particularly within the subset of patients with failing Fontan physiology, appears appropriate, although presently it is institution dependent $[2,3]$.

An increasing number of patients with Fontan physiology are surviving well into adulthood and are at risk of hepatic sequelae. The most effective method of surveillance for liver disease in Fontan patients remains to be clearly defined. The unfortunate outcome and early death of the patient in this case illustrates the importance of doing so, particularly when patients exhibit signs of hepatic congestion.

\section{Acknowledgement}

We acknowledge Dr. Paul Naylor's efforts in facilitating the construction of this report.

\section{Statement of Ethics}

This case report is in compliance with the guidelines for human studies and in accordance with the World Medical Association Declaration of Helsinki. The subject of this case has not been referred to be her real name, and further no identifying image has been included with this case. 


\section{Disclosure Statement}

The authors have no conflicts of interest to declare.

\section{Funding Sources}

No external funding was utilized in the creation of this case report.

\section{Author Contributions}

Conceptualization, M.E., A.D., S.J., B.P.R.; investigation, B.P.R., A.D., S.B.; supervision, M.E., S.J; writing - original draft, A.D., B.P.R.; writing - review and editing, M.E., B.P.R.

\section{References}

1 Egbe A, Uppu S, Lee S, Ho D, Srivastava S. Changing prevalence of severe congenital heart disease: a population-based study. Pediatr Cardiol. 2014 Oct;35(7):1232-8.

2 Josephus Jitta D, Wagenaar LJ, Mulder BJ, Guichelaar M, Bouman D, van Melle JP. Three cases of hepatocellular carcinoma in Fontan patients: review of the literature and suggestions for hepatic screening. Int J Cardiol. 2016 Mar;206:21-6.

3 Hilscher MB, Johnson JN, Cetta F, Driscoll DJ, Poterucha JJ, Sanchez W, et al. Surveillance for liver complications after the Fontan procedure. Congenit Heart Dis. 2017 Mar;12(2):124-32.

4 Camposilvan S, Milanesi O, Stellin G, Pettenazzo A, Zancan L, D’Antiga L. Liver and cardiac function in the long term after Fontan operation. Ann Thorac Surg. 2008 Jul;86(1):177-82.

5 Atluri P, Gaffey A, Howard J, Phillips E, Goldstone AB, Hornsby N, et al. Combined heart and liver transplantation can be safely performed with excellent short- and long-term results. Ann Thorac Surg. 2014 Sep;98(3):858-62.

6 Daniels CJ, Bradley EA, Landzberg MJ, Aboulhosn J, Beekman RH 3rd, Book W, et al. Fontan-Associated Liver Disease: Proceedings from the American College of Cardiology Stakeholders Meeting, October 1 to 2, 2015, Washington DC. J Am Coll Cardiol. 2017 Dec;70(25):3173-94.

7 Gordon-Walker TT, Bove K, Veldtman G. Fontan-associated liver disease: A review. J Cardiol. 2019 Mar;S0914-5087(19)30059-0.

8 Ohashi K, Iimuro Y, Suzumura K, Hai S, Tanaka S, Hirano T, et al. Development of hepatocellular carcinoma in cardiac congestive liver fibrosis: report of a case. Clin J Gastroenterol. 2015 Jun;8(3):143-7.

9 Wolf JH, Sulewski ME, Cassuto JR, Levine MH, Naji A, Olthoff KM, et al. Simultaneous thoracic and abdominal transplantation: can we justify two organs for one recipient? Am J Transplant. 2013 Jul;13(7):1806-16.

10 Diamond T, Ovchinsky N. Fontan-associated liver disease: monitoring progression of liver fibrosis. Clin Liver Dis (Hoboken). 2018 Jan;11(1):1-5.

11 Téllez L, Rodriguez-Santiago E, Albillos A. Fontan-Associated Liver Disease: A Review. Ann Hepatol. 2018;17(2):192-204.

12 Kutty SS, Peng Q, Danford DA, Fletcher SE, Perry D, Talmon GA, et al.; Liver Adult-Pediatric-CongenitalHeart-Disease Dysfunction Study (LADS) Group. Increased hepatic stiffness as consequence of high hepatic afterload in the Fontan circulation: a vascular Doppler and elastography study. Hepatology. 2014 Jan;59(1):251-60.

13 Poterucha JT, Johnson JN, Qureshi MY, O'Leary PW, Kamath PS, Lennon RJ, et al. Magnetic Resonance Elastography: A Novel Technique for the Detection of Hepatic Fibrosis and Hepatocellular Carcinoma After the Fontan Operation. Mayo Clin Proc. 2015 Jul;90(7):882-94. 


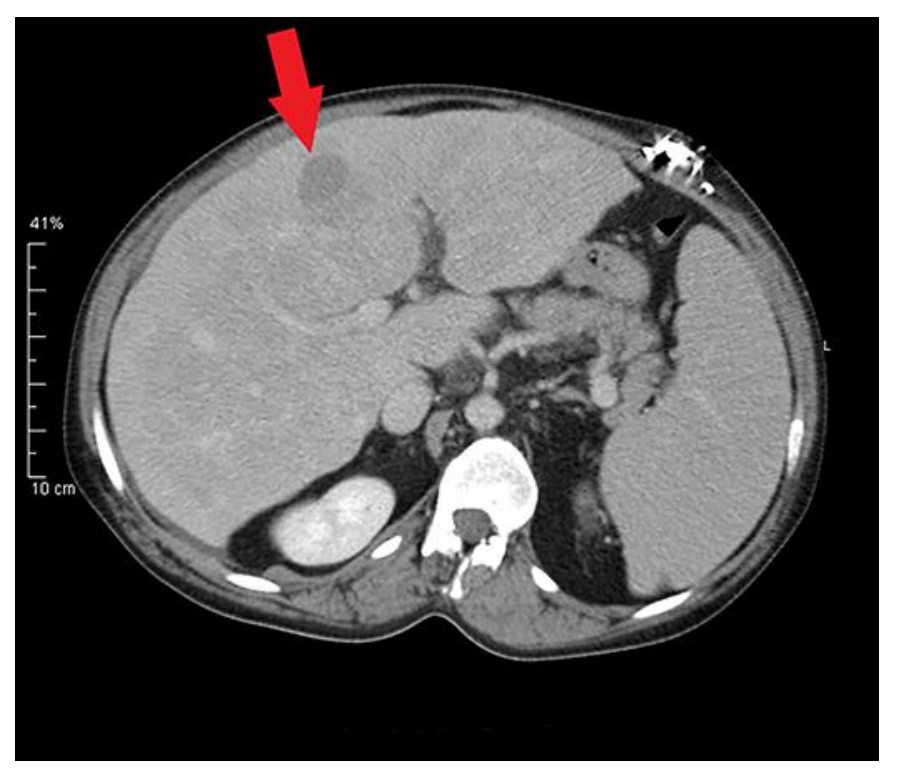

Fig. 1. CT scan of the abdomen with a red arrow specifying the location of a mass that enhanced during late arterial phase and then washed out rapidly in the portal venous phase, compared to the rest of the liver. 